\title{
Light scattering by lithium tantalate at room temperature
}

\author{
A. F. Penna, A. Chaves, * P. da R. Andrade, and S. P. S. Porto \\ Physics Department, University of Southern California, Los Angeles, California 90024 \\ and Instituto de Física "Gleb Wataghin," Universidade Estadual de Campinas, 13100, Campinas, SP, Brazil
}

(Received 3 September 1975)

\begin{abstract}
The room-temperature Raman spectra of $\mathrm{LiTaO}_{3}$ for all symmetry phonons are investigated. We make a new assignment of the phonons, which agrees with the selection rules of a $C_{3}$ symmetry instead of $C_{3 v}$, contrary to Kaminow and Johnston's previous work. The oblique phonon dispersions are obtained and turn out to be consistent with our phonon assignments. We obtain also the polariton dispersion curve and, from it, the lowfrequency dielectric constant.
\end{abstract}

\section{INTRODUCTION}

Lithium tantalate $\left(\mathrm{LiTaO}_{3}\right)$ was found to be ferroelectric in 1949 by Mathias and Remeika, ${ }^{1}$ and also to exhibit a large electro-optic effect by Lenzo et $a l .^{2}$ With the production today of high-quality single crystals of $\mathrm{LiTaO}_{3}$ a great deal of interest in this material has been revived for use in electro-optic devices as well as in nonlinear optical experiments. The room-temperature crystal structure has been studied by $\mathrm{x}-\mathrm{ray}^{3}$ and neutron diffraction ${ }^{4}$ and they all agree that it is uniaxial trigonal belonging to the point group $C_{3 v}$. Ball$\operatorname{man}^{5}$ showed that the properties of many ferroelectrics have a marked dependence on the melt stoichiometry variations and particularly for $\mathrm{LiTaO}_{3}$ the Curie temperature may change from 540 to $700^{\circ} \mathrm{C}$, depending on the growth conditions. Above $T_{c}$ it is paraelectric and has a $D_{3 d}$ pointgroup symmetry. ${ }^{3,4}$

In our work we performed a complete Ramanscattering analysis of $\mathrm{LiTaO}_{3}$ single crystal at both room and high temperature ${ }^{6}$ We propose a new assignment of the normal modes, in disagreement with the works by Kaminow and Johnston ${ }^{7}$ and Barker et $a l .,^{8}$ and study consistently the oblique phonon dispersion; we also investigate the polariton dispersion and from the data we evaluate the low-frequency dielectric constant.

\section{THEORY}

The crystal structure of $\mathrm{LiTaO}_{3}$ has been studied recently by several authors. ${ }^{3,4}$ They all agree that its ferroelectric phase belongs to the point group $C_{3 v}$ with two molecules per unit cell. This implies a total of 30 degrees of freedom, and the irreducible representation for the vibrational modes are given by

$$
4 A_{1}(z)+9 E(x)+9 E(y)+5 A_{2},
$$

where the $A_{1}$ and $E$ modes are both Raman and infrared active, whereas the $A_{2}$ 's are inactive in
Raman and infrared. The Raman tensors associated with the active modes $\operatorname{are}^{9}$

$$
\begin{aligned}
& A_{1}(z)=\left(\begin{array}{lll}
a & & \\
& a & \\
& & b
\end{array}\right), \\
& E(y)=\left(\begin{array}{ccc}
c & 0 & 0 \\
0 & -c & d \\
0 & d & 0
\end{array}\right), \\
& E(-x)=\left(\begin{array}{ccc}
0 & -c & -d \\
-c & 0 & 0 \\
-d & 0 & 0
\end{array}\right) .
\end{aligned}
$$

The letters in parentheses indicate the direction of the polarization associated with the modes, with respect to the crystallographic axis.

Since all the 13 modes which are Raman active carry polarization, their interaction with the macroscopic field produced by the infrared-active phonons may be able to raise the degeneracy between the longitudinal (LO) and transverse (TO) modes, doubling the number of lines in the Raman scattering spectrum. The term "doubling" should be understood with reserve because the degeneracy between the LO and TO's may sometimes be too small to show a detectable splitting in the spectrum.

Before presenting the experimental data and discussing the results, we would like to discuss the possibility of a small distortion in the unit cell that can cause a lowering in the effective symmetry. This possibility is not academic but it is supported in the experimental results, as we shall see later. Figure 1 displays the unit cell of $\mathrm{LiTaO}_{3}$. If by any chance a small distortion in the oxygen triangles breaks down the reflection symmetry, the new point group will be $C_{3}$ instead of $C_{3 v}$. Even if in the average the crystal as a whole shows a $C_{3 v}$ symmetry, the response of the unit 


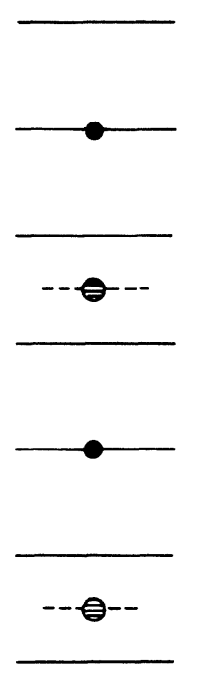

(a)

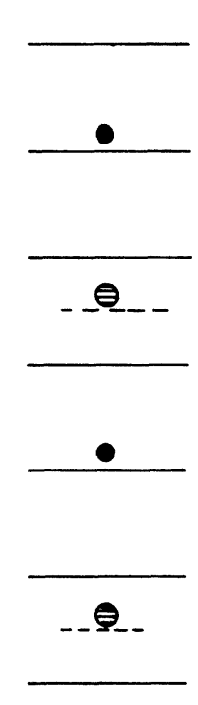

(b)

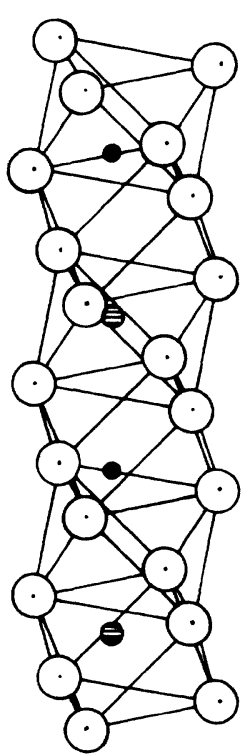

(c)
FIG. 1. Structure of lithium tantalate: (c) spatial structure of the unit cell, open circles- $\mathrm{O}$, solid circles-Li, cross-hatched-Ta; (b) model of the ferroelectric phase; (a) model of the paraelectric phase.

cell will be that of a $C_{3}$ symmetry. Looking at the correlation table of the $C_{3 v}$ group and its subgroup ${ }^{10}$

\begin{tabular}{c|cc}
$C_{3 v}$ & $C_{3}$ & $C_{s}$ \\
\hline$A_{1}$ & $A$ & $A^{\prime}$ \\
$A_{2}$ & $A$ & $A^{\prime \prime}$ \\
$E$ & $E$ & $E^{\prime}+A^{\prime \prime}$
\end{tabular}

we see that the five $A_{2}$ inactive modes may become $A_{1}$ active with the same Raman tensor as $A_{1}$ given above. If that small distortion is not uniform in the whole crystal, we expect a very weak intensity in the spectrum for the five extra modes, with no TO-LO splitting since the $A_{2}$ modes carry no polarization.

In the paraelectric phase $\mathrm{LiTaO}_{3}$ is $\mathrm{known}^{3,4}$ to be centrosymmetric with $D_{3 d}$ point-group symmetry, with the following irreducible representation, for the optical modes:

$$
A_{1 g}+3 E_{g}+3 A_{2 u}(z)+2 A_{1 u}+3 A_{2 g}+5 E_{u}(x)+5 E_{u}(y),
$$

where $A_{1}$ and $E_{g}$ are Raman active, $A_{2 u}$ and $E_{u}$ are infrared active, and the others are inactive in both.

The static dielectric constant $\epsilon_{0}$ can be evaluated by means of the generalized Lyddane-Sachs-Teller relation ${ }^{11}$

$$
\epsilon_{0}=\epsilon_{\infty} \prod_{i}\left(\frac{\omega_{\mathrm{LO}}^{i}}{\omega_{\mathrm{TO}}^{i}}\right)^{2},
$$

where $\epsilon_{\infty}$ is the high-frequency dielectric constant, $\omega_{\text {LO }}^{i}$ and $\omega_{\text {ro }}^{i}$ are the longitudinal and transverse phonon frequency, respectively, and the product is to be taken over all the polar modes along one of the axes. We want to point out that expression (1) was derived assuming all the modes in the crystal are harmonic oscillators with no damping consideration. Nevertheless, Cochran and Cowley ${ }^{11}$ have estimated that any deviation from relation (1) for real crystals, owing to the anharmonicity, should be no larger than $5 \%$.

The properties of lattice vibrations that carry an electric moment differs significantly from those of nonpolar vibrations, in the sense that the radiation field of polar vibrations in the crystal couples to the mechanical vibrations to create a photon-phonon mixed excitation called polariton. Huang, in $1951,{ }^{12}$ showed that in the whole range of frequency $\omega$ and wave vector $q$ the dispersion relation is given by

$$
\omega^{2} \epsilon(\omega)=c^{2} q^{2},
$$

where the frequency-dependent dielectric constant $\epsilon(\omega)$ is given by ${ }^{13}$

$$
\epsilon(\omega)=\epsilon_{\infty}+\sum_{j=1}^{N} \frac{S_{j} \omega_{j}^{2}}{\omega_{j}^{2}-\omega^{2}+i \Gamma_{j} \omega} .
$$

$\Gamma_{j}$ is the damping factor associated with the transverse phonon frequency $\omega_{j}, \epsilon_{\infty}$ is the high-frequency dielectric constant, and $S_{j}$ the dielectric transition strength.

From expression (3) we see that for very small $\omega, \epsilon(\omega)$ has very slight dependence on $\omega$ and its value approaches $\epsilon_{0}$, so that relation (2) tells us that in this limiting case $\omega$ and $q$ are related to each other through a linear function, and can be written

$$
\omega / q=1 / \sqrt{\epsilon_{0}} .
$$

This last relation shows that we can evaluate the low-frequency dielectric constant from the lower polariton dispersion curve slope, in the limit where $\omega$ (or $q$ ) goes to zero. This fact has been used lately ${ }^{14,15}$ as an alternative way to study the dielectric properties of crystals.

\section{EXPERIMENTS}

Our experiments were done in a large poled single crystal of $\mathrm{LiTaO}_{3}$, provided to us by $\mathrm{Dr}$. A. A. Ballman, from Bell Labs., with a ferroelectric Curie temperature of $625^{\circ} \mathrm{C}$. The crystal was oriented by Laue back-reflection $\mathrm{x}$-ray photographs and cut with faces perpendicular to the crystallographic axis, to within $1^{\circ}$ precision. This allowed scattering symmetry for almost pure TO 
or LO. All the Raman experiments described in this chapter were obtained at room temperature, using the 5145- $\AA$ line of a CRL model 52 argon-ion laser, a Spex double monochromator model 1401, a photomultiplier, and a dc recording. The slit was selected in order to give us a resolution smaller than $1 \mathrm{~cm}^{-1}$.

To represent the scattering geometry and the polarization used in the measurements, we use the standard Raman notation $k(i j) q$, where $k$ is the propagation direction of the incident light polarized along $i$, and $q$ is the propagation direction of the scattered light polarized along $j$. Sometimes we also use the notation $k(i j) k+\Delta l$, where $l$ can be either $i$ or $j$, and by that we mean that the light was collected in forward direction through two small holes displaced along $l$ direction, as shown in the diagram (a) of Fig. 2. Both $E$ and $A_{1}$ phonons were measured in every possible geometry to search for any $k$ dependence, as well as all the Raman tensor components in order to improve the assignment of the weakest phonons. We were also very careful in choosing the propagation direction of the incident and scattered radiations, as well as its polarizations, in order to obtain data very close to pure-LO and to pure-TO phonons. However, owing to the high index of refraction of the crystal (2.22), we could not use any liquid matching to avoid internal reflection, so that its reflex is always present in the backward and forward scattering.

The fraction of the light reflected internally is given by

$$
R=[(n-1) /(n+1)]^{2},
$$

where $n$ is the index of refraction at the laser frequency. For $\mathrm{LiTaO}_{3}, n=2.22$, and this value into Eq. (5) tells us that about $14 \%$ of the incident light is reflected inside the crystal. The reflected and incident light generate phonons propagating in different direction and this may give rise, sometimes, to a mixing of the transverse and longitudinal modes in the spectra, mainly for those having a very strong Raman activity.

The oblique-phonon measurements were done using the setup shown in Fig. 2(a). By rotating the stopper through its center, we can select phonons propagating in any direction in the plane $Z Y$. In this way, with a simple arrangement, we can easily follow the oblique-phonon dispersion, without changing the experimental geometry and keeping a more consistent relation between the phonon intensities for all the angles.

The $A_{1}$ symmetry polaritons were studied at the configuration $X(Z Z) X+\Delta Y$ and the wave vector $q$ were selected by using a stopper, shown in Fig. 2,
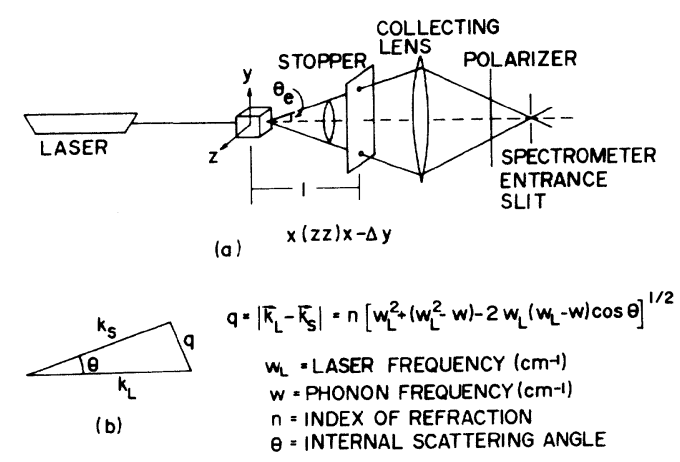

FIG. 2. Scattering geometry for polariton and obliquephonon experiments (a), and diagram for the momentum conservation law (b).

with two holes oriented along the $Y$ axis. The wave vector is a function of the angle $\theta$ and it can be changed by changing either the distance between the holes in the stopper or by changing the distance from the stopper to the crystal. In this way we were able to obtain as low as $300 \mathrm{~cm}^{-1}$ for the $q$ value. The scattering angle can be measured with a high degree of precision and an error of only $5 \%$, or less, is to be expected for angles not smaller than $0.5^{\circ}$. Observations were made in two different poled crystals and no appreciable changes were detected in the spectra. However, we tried to measure the polaritons in a third, unpoled crystal, and could not observe any dispersion at all, even for very small $q$ (smaller than $300 \mathrm{~cm}^{-1}$ ). We believe this phenomenon is related to the domains inside the crystal and it will be discussed in more detail later.

\section{PHONONS AND OBLIQUE PHONONS}

The optical modes in $\mathrm{LiTaO}_{3}$ have been studied before by Barker et al. ${ }^{8}$ in 1970 using infrared reflectivity and also by Kaminow and Johnston ${ }^{7,16}$ in 1967 using Raman spectra. For later reference, their results are tabulated in Tables I-IV.

The $A_{1}$ phonons taken at the configuration $X(Z Z) Y$ for the TO's and $X(Z Z) X+\Delta Z$ for the LO's are shown in Figs. 3 and 4 . In Fig. 3 we see already the evidence of seven peaks, instead of four predicted by group theory for the $C_{3 v}$ symmetry. All those phonons, but the one at $186 \mathrm{~cm}^{-1}$, have been observed by Kaminow and Johnston ${ }^{7}$ who chose four $\left(201,253,356\right.$, and $\left.600 \mathrm{~cm}^{-1}\right)$ as the fundamental modes, and the additional structures are attributed to coupling between the $A_{1}$ and $E$ modes, as a consequence of a strain perturbation. Although the mode at $186 \mathrm{~cm}^{-1}$ has not been mentioned in Ref. 13, it seems to us to be a fundamental one. It is evident from both polariton and oblique-phonon dispersion shown in Figs. 5 and 6 , that is, the pho- 
TABLE I. E-symmetry phonon parameters observed by Raman spectra, from Ref. 13 .

\begin{tabular}{ccc}
\hline \hline TO $\left(\mathrm{cm}^{-1}\right)$ & LO $\left(\mathrm{cm}^{-1}\right)$ & $\Gamma\left(\mathrm{cm}^{-1}\right)$ \\
\hline 75 & 80 & 9.5 \\
140 & 163 & 3 \\
206 & 248 & 3 \\
251 & 278 & 5 \\
316 & 318 & 3 \\
383 & 452 & 6 \\
462 & 474 & 3 \\
596 & 648 & 5.5 \\
662 & 870 & 7.5 \\
\hline \hline
\end{tabular}

non going to zero in the polariton dispersion, while the one at $202 \mathrm{~cm}^{-1}$ stays there; in the obliquephonon both modes disperse but in opposite directions, making it clear that we have, in fact, two $A_{1}$ (TO) phonons in that region instead of one reported before. ${ }^{7,8}$

If we were to attribute the additional structure in the transverse $A_{1}$ spectra to strains, which may cause an $E$ mode to become Raman active for a $(Z Z)$ filter, we expect those peaks to be much weaker than the fundamental $E$-type modes originating them. What happens is exactly the contrary, the additional lines in the $A_{1}$ (TO) spectra are much stronger than those around the same frequency in the $E$ spectra. We see no choice other than to accept every peak as a fundamental $A_{1}$-type mode.

A look at the spectrum $Z(X X) \bar{Z}$ in Fig. 7, which should give us $E(\mathrm{TO})+A_{1}(\mathrm{LO})$, shows a new phonon at $751 \mathrm{~cm}^{-1}$. Although it is very weak in the $(Z Z)$ configuration and is not visible in Fig. 3, we can see it clearly if we enlarge the spectrum about

TABLE II. $A_{1}$-symmetry phonon parameters observed by Raman spectra, from Ref. 13 .

\begin{tabular}{ccc}
\hline TO $\left(\mathrm{cm}^{-1}\right)$ & LO $\left(\mathrm{cm}^{-1}\right)$ & $\Gamma\left(\mathrm{cm}^{-1}\right)$ \\
\hline 201 & 245 & 7.5 \\
253 & 347 & 7.5 \\
356 & 399 & 4 \\
600 & 864 & 4 \\
\hline
\end{tabular}

TABLE III. $E$-symmetry phonon parameters of $\mathrm{LiTaO}_{3}$ determined by infrared reflectivity, from Ref. 12 .

\begin{tabular}{ccc}
\hline \hline TO $\left(\mathrm{cm}^{-1}\right)$ & LO $\left(\mathrm{cm}^{-1}\right)$ & $\Gamma\left(\mathrm{cm}^{-1}\right)$ \\
\hline 142 & & 14 \\
$(165)^{\mathrm{a}}$ & $(165)$ & 11 \\
$(175)$ & $(175)$ & 7 \\
& 192 & 13 \\
$(215)$ & $(215)$ & 19 \\
$(238)$ & $(238)$ & 9 \\
253 & & \\
& 278 & 14 \\
316 & & 26 \\
& 345 & 24 \\
375 & & \\
$(405)$ & $(405)$ & 6 \\
462 & 455 & 32 \\
& & 34 \\
594 & 474 & 22 \\
$(673)$ & $(673)$ & $(750)$ \\
$(750)$ & 855 & \\
\hline \hline
\end{tabular}

${ }^{a}$ Bracketed modes are very weak in reflectivity.

ten times. So we decided to assign it as an $A_{1}$ mode. Figure 5 shows the evidence of another $A_{1}$ (TO) at about $460 \mathrm{~cm}^{-1}$ dispersing in the obliquephonon with the $A_{1}(\mathrm{LO})$ at $401 \mathrm{~cm}^{-1}$. The seven phonons we started with plus those two we just mentioned, makes a total of nine $A_{1}$ (TO) modes, some of them very weak as we have noted, but still very clearly belonging to the $A_{1}$ symmetry.

These extra phonons assigned as $A_{1}$ type can be understood if some kind of disorder lowers the symmetry of the crystal from $C_{3 v}$ to $C_{3}$. In this case, according to the correlation table for the $C_{3 v}$ point group discussed in Sec. II, the $A_{2}$ inactive modes transform into $A_{1}$ and become active. Looking at the unit cell of $\mathrm{LiTaO}_{3}$ in Fig. 1, this hypothesis seems very reasonable if we consider that the structure of oxygens, upon which

TABLE IV. $A_{1}$-symmetry phonon parameters of $\mathrm{LiTaO}_{3}$ determined by infrared reflectivity, from Ref. 12 .

\begin{tabular}{ccc}
\hline \hline TO $\left(\mathrm{cm}^{-1}\right)$ & LO $\left(\mathrm{cm}^{-1}\right)$ & $\Gamma\left(\mathrm{cm}^{-1}\right)$ \\
\hline 200 & 241 & 28 \\
241 & 345 & 30 \\
357 & 388 & 11 \\
596 & 625 & 18 \\
657 & $(760)^{\mathrm{a}}$ & 56 \\
760 & 862 & 44 \\
\hline \hline
\end{tabular}

${ }^{a}$ Bracketed modes are very weak in reflectivity. 


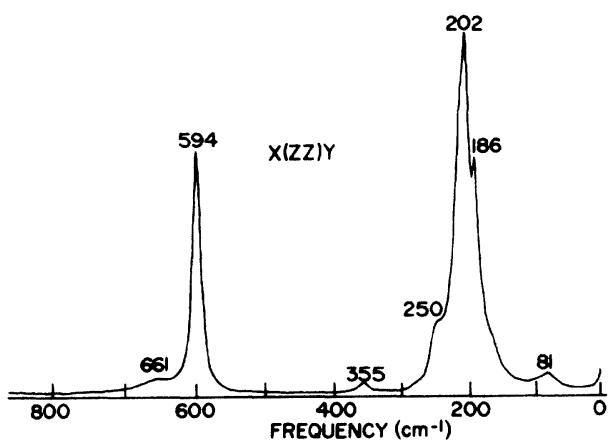

FIG. 3. ( $Z Z)$ spectrum of the $A_{1}(\mathrm{TO})$ phonons in $\mathrm{LiTaO}_{3}$.

the unit cell is based, may not be very well aligned to allow a reflection plane. A small distortion in an oxygen layer, with respect to the next one, may break down the reflection symmetry and the crystal becomes $C_{3}$.

According to our interpretation, all the nine phonons observed should be accepted as fundamental $A_{1}$ type and the crystal point group as $C_{3}$. To reinforce our point of view we want to point out that Barker et al., ${ }^{8}$ to fit his infrared reflectivity with classical oscillators, needed at least six $A_{1}$ phonons (see Table IV).

The $A_{1}(\mathrm{LO})$ taken at $X(Z Z) X+\Delta Z$ is shown in Fig. 4, where we indicate with an asterisk the peaks coming from other symmetries, all of them consequences of the scattering geometry that pick up slightly oblique phonons instead of pure LO. If we compare this spectrum with those for the $A$ oblique phonon (Figs. 5 and 8 ) we can see that it corresponds to $\Phi$ around $10^{\circ}$. Of the two peaks around $580 \mathrm{~cm}^{-1}$, one is a polariton of the oblique phonon and the other is the TO created by back reflection inside the crystal. The peak at $419 \mathrm{~cm}^{-1}$ does not correspond to the exact position of the LO, but to an oblique phonon whose branch goes from $458 \mathrm{~cm}^{-1}$ (TO) to $401 \mathrm{~cm}^{-1}$. Its exact frequency is $401 \mathrm{~cm}^{-1}$, as shown in Fig. 7. This completes our discussion for the $A_{1}$ modes and the final assignment is in Table $\mathrm{V}$.

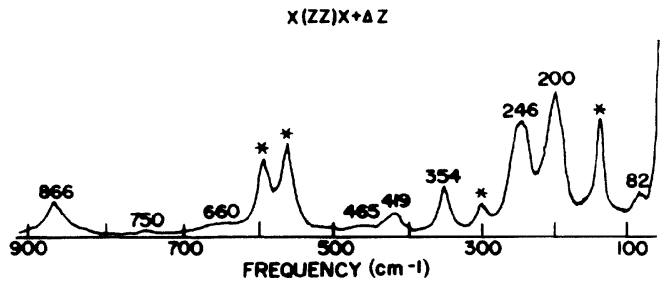

FIG. 4. (ZZ) spectrum of the $A_{1}$ (LO) phonons in $\mathrm{LiTaO}_{3}$. The lines indicated with an asterisk are spillover from other symmetries.

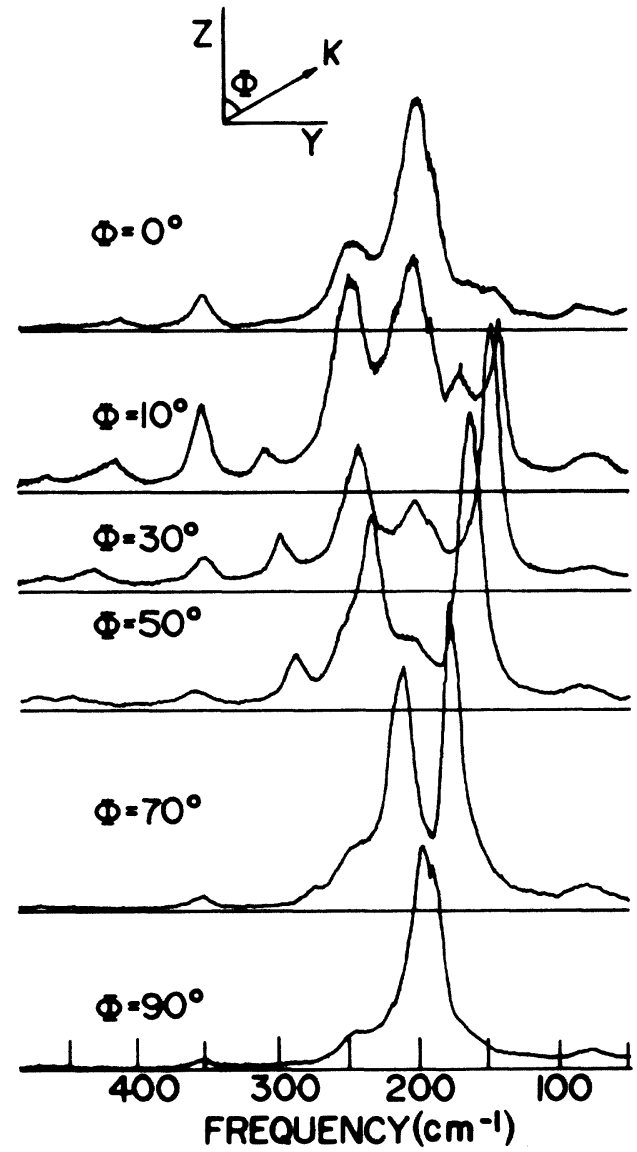

FIG. 5. Low-frequency $(Z Z)$ spectra of the $A_{1}$ oblique phonons. $\phi=0^{\circ}$ correspond, to pure LO and $\phi=90^{\circ}$ to pure TO phonons.

The $E$ phonons, polarized in the $X Y$ plane, are shown in Figs. 9 and 10, taken in the configurations $Z(X Z) Y$ and $X(Z Y) X+\Delta Y$. The $E(\mathrm{TO})$ spectra (Fig. 9) shows nine peaks; one of them has been assigned by us as a spill over (asterisk) (namely, the one at $200 \mathrm{~cm}^{-1}$ ) to make it consistent with the oblique-phonon dispersion, to be discussed in Sec. V. This strong spill over seems reasonable if we bear in mind that at that position two strong $A_{1}$ (one TO and one LO), and one strong $E(\mathrm{LO})$ are present.

In the beginning we doubted the assignment of the structure around $70 \mathrm{~cm}^{-1}$ (see Fig. 9) as a phonon. We thought first of a spill over of the $A_{1}$ modes at the same frequency or even a secondorder broad band. However, if we investigate that region in more detail, we see clearly the existence of two peaks (see Fig. 11); one is a true $E$ (TO) at $69 \mathrm{~cm}^{-1}$ and the second peak is a spill over of the (much stronger) $A_{1}$ at $81 \mathrm{~cm}^{-1}$.

If we disregard the peak at $200 \mathrm{~cm}^{-1}$ in the $E$ (TO) spectra (Fig. 9) we need one more phonon 


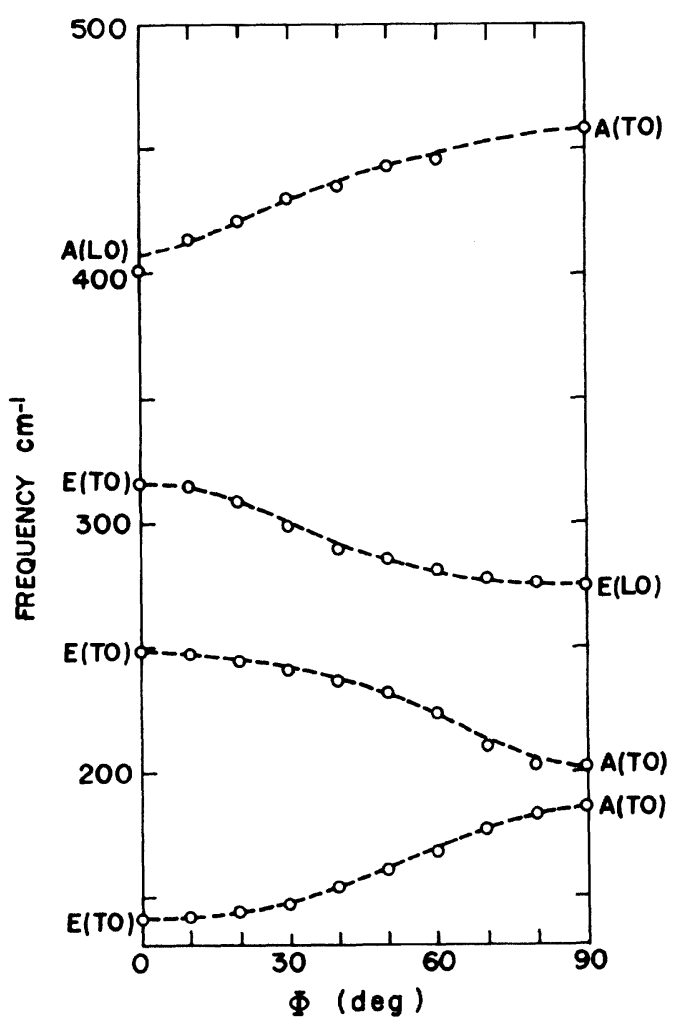

FIG. 6. Oblique phonon dispersion curves of the most dispersive modes in $\mathrm{LiTaO}_{3}$.

to complete the nine predicted by group theory. Though we could not find the missing phonon in the appropriate $E$ (TO) configuration, because it is very weak, its existence becomes evident from the oblique-phonon dispersion shown in Fig. 12, where it disperses with the $E(\mathrm{LO})$ at the same frequency $\left(345 \mathrm{~cm}^{-1}\right)$.

Figure 10 shows the $E$ (LO) spectrum taken at $X(Z Y) Z+\Delta Y$, where eight peaks are indicated as phonons and four as spill over (asterisk). The ninth missing phonon is a very weak one, at 470

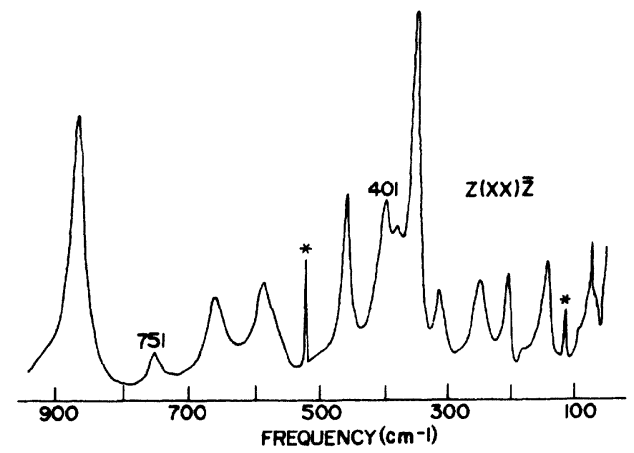

FIG. 7. (XX) Raman spectrum of $\mathrm{LiTaO}_{3}$, showing $A_{1}(\mathrm{LO})+E(\mathrm{TO})$.

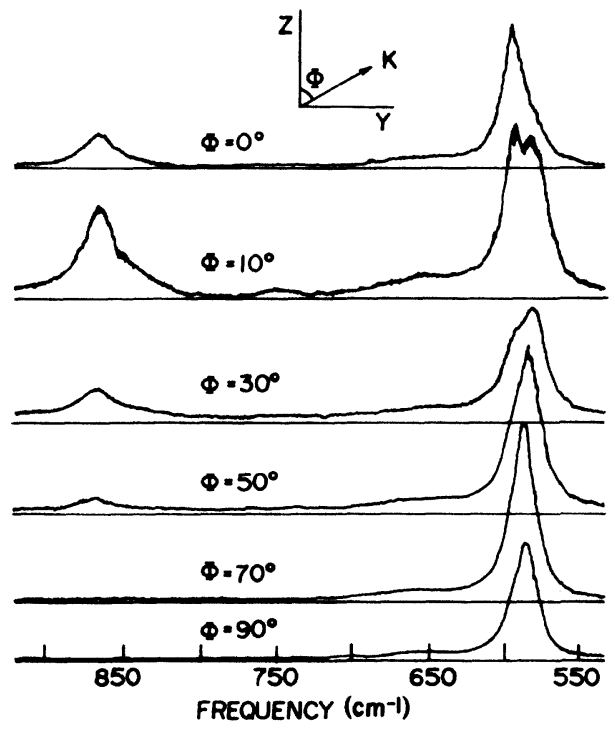

FIG. 8. High-Frequency $(Z Z)$ spectra of the $A_{1}$ oblique phonons.

$\mathrm{cm}^{-1}$, and disperses with the $A(\mathrm{LO})$ at $465 \mathrm{~cm}^{-1}$. That is the only place where we can track it (Fig. $5)$. Three out of the four spill over $(143,316$, and $590 \mathrm{~cm}^{-1}$ ) are a result of the internal back reflection, giving origin to a scattering of the type $X(Z Y) \bar{X}$ which activates the $E(\mathrm{TO})$ phonons, and the peak at $186 \mathrm{~cm}^{-1}$ is a consequence of the slightly oblique symmetry of the scattering which can be checked with Fig. 12. Our final assignment for the $E$ phonons is given in Table VI.

Assuming the crystal has $C_{3}$ symmetry with nine $E$ and nine $A_{1}$ Raman active modes, we can evalu-

TABLE V. $A_{1}$-symmetry phonon parameters observed by Raman spectra, from this work.

\begin{tabular}{ccc}
\hline \hline TO $\left(\mathrm{cm}^{-1}\right)$ & LO $\left(\mathrm{cm}^{-1}\right)$ & $\Gamma\left(\mathrm{cm}^{-1}\right)$ \\
\hline 81 & 82 & 21 \\
186 & 200 & 15 \\
202 & 246 & 26 \\
250 & 354 & 24 \\
355 & 401 & 13 \\
458 & 465 & 13 \\
594 & 660 & 14 \\
661 & 750 & 29 \\
751 & 866 & 29 \\
\hline \hline
\end{tabular}




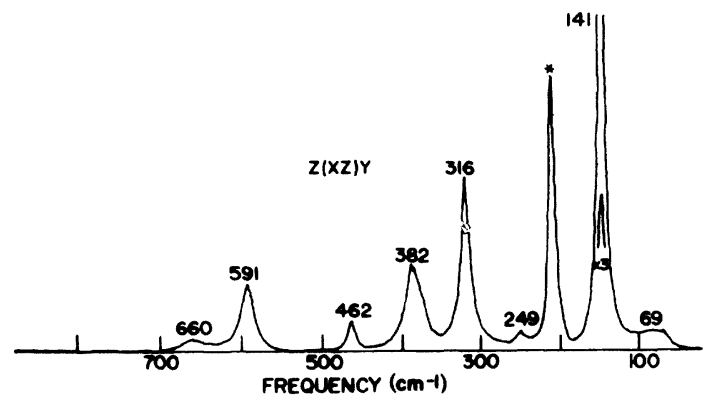

FIG. 9. $(X Z)$ spectrum of the $E(\mathrm{TO})$ phonons in $\mathrm{LiTaO}_{3}$. The line indicated with an asterisk is spillover from other symmetries.

ate the low-frequency dielectric constant using the Lyddane-Sachs-Teller relation. With $\epsilon_{\infty}=4.9,{ }^{17}$ we obtain from Eq. (1) the following results:

$\epsilon_{0}=48$ in the $Z$ direction

and

$\epsilon_{0}=57$ in the $X Y$ plane.

Yamada et $a l .^{18}$ measured the values 46 and 53 , respectively, using an admittance bridge at 10 $\mathrm{kHz}$, which are in fairly good agreement (less than $7 \%$ ) with our results.

The oblique phonons for $A_{1}$ symmetry were studied in the $X(Z Z) X+\Delta \Phi$ configuration, where $\Delta \Phi$ represents the phonon propagation direction with respect to the crystal $Z$ axis. For $\Phi=90^{\circ}$ the phonon is propagating along $Y$ axis and we are collecting pure TO; for $\Phi=0^{\circ}$, the propagation direction is $Z$, giving a LO symmetry. The spectra were taken for each $5^{\circ}$ interval between $0^{\circ}$ and $90^{\circ}$ and are shown in Figs. 5 and 8 for six different angles.

The first $A_{1}$ (TO) at $81 \mathrm{~cm}^{-1}$ has a dispersion with the first $A_{1}(\mathrm{LO})$ at $82 \mathrm{~cm}^{-1}$, while the second pair of TO's, 186 and $202 \mathrm{~cm}^{-1}$, disperses in opposite directions; one to the $E$ (TO) at $141 \mathrm{~cm}^{-1}$ and the other to the $A_{1}(\mathrm{LO})$ at $246 \mathrm{~cm}^{-1}$. When the two peaks start splitting apart and they become

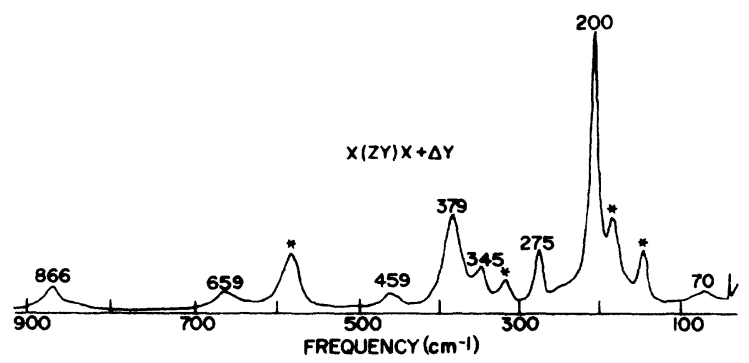

FIG. 10. $(Z Y)$ spectrum of the $E(L O)$ phonons in $\mathrm{LiTaO}_{3}$. The lines indicated with an asterisk spillover from other symmetries.

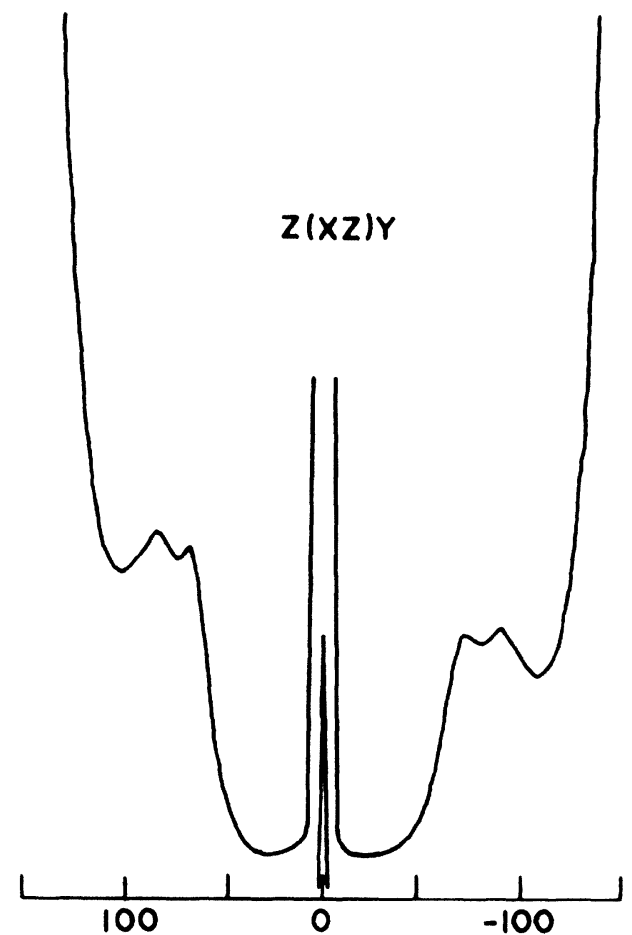

FIG. 11. (XZ) spectrum of the $E(T O)$ phonons. This spectrum is the same shown in Fig. 9, enlarged 10 times.

more pure LO, the $A_{1}(\mathrm{LO})$ at $200 \mathrm{~cm}^{-1}$ is enhanced showing that it must disperse with the $E(\mathrm{LO})$ at the same frequency. Since the $A_{1}$ (TO) at $202 \mathrm{~cm}^{-1}$ disperses to the $A_{1}(\mathrm{LO})$ at $246 \mathrm{~cm}^{-1}$, the $A_{1}$ (TO) at $250 \mathrm{~cm}^{-1}$, visible in the spectra until $50^{\circ}$, has to go to the $E(T O)$ at $249 \mathrm{~cm}^{-1}$. The next two $A_{1}$ (TO)'s at 355 and $458 \mathrm{~cm}^{-1}$ go to $A_{1}$ (LO)'s at 354 and 401 , respectively (Fig. 5).

The last part of the spectra are shown in Fig. 8, and they are about the same for both $A_{1}$ and $E$ phonons. At a glance it looks like the phonon at $594 \mathrm{~cm}^{-1}$ is changing position when we go from TO to LO configuration. In fact, what is happening is the following: in the TO symmetry, what we see is the polariton of the phonon at lower frequency, whose intensity goes to zero when the spectra becomes more and more pure LO, and the consequence of the back reflection inside the crystal (giving pure TO) starts enhancing in the spectra until $\Phi=0^{\circ}$, where it dominates completely. This will become more clear if we remember that the spectra for $70^{\circ}, 50^{\circ}$, and $30^{\circ}$ are three times enlarged with respect to that at $90^{\circ}$, while the two remaining spectra at $10^{\circ}$ and $0^{\circ}$ are enlarged about ten times. The last phonon, nonexistent in the $A_{1}$ (TO) symmetry, starts to become clear as the spectra changes from $T O$ to LO. For the $E$ phonon the same thing happens. This made us believe that the $A_{1}(\mathrm{LO})$ at $866 \mathrm{~cm}^{-1}$ 


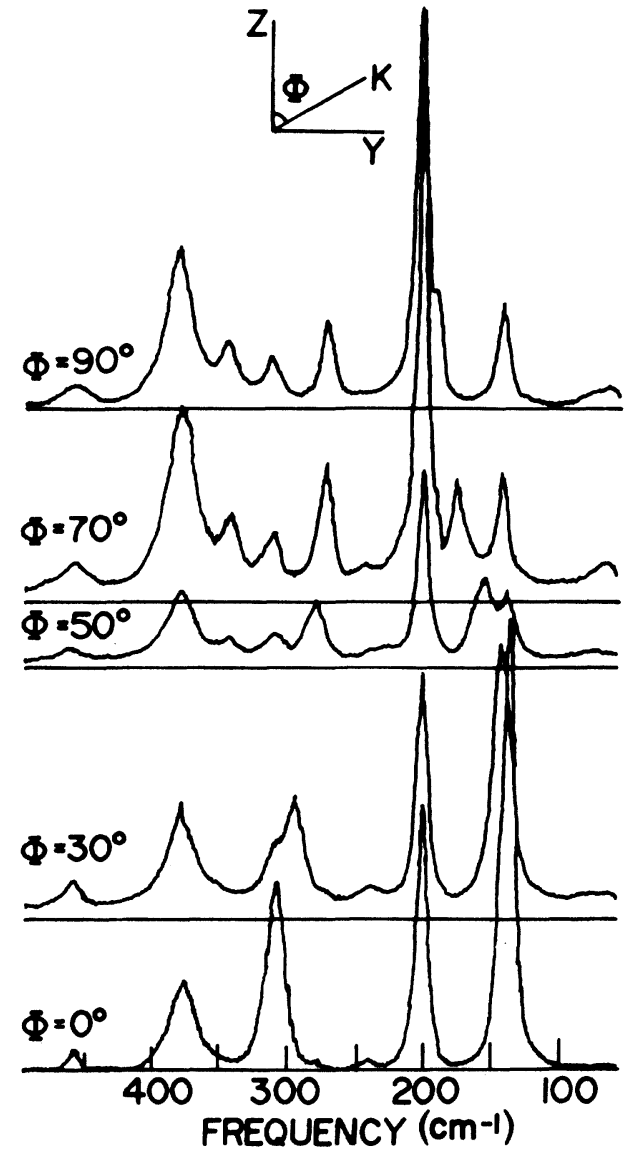

FIG. 12. (YZ) spectra of the $E$ oblique phonons. $\phi=0^{\circ}$ corresponds to pure TO and $\phi=90^{\circ}$ to pure LO phonons.

TABLE VI. $E$-symmetry phonon parameters observed by Raman spectra, from this work.

\begin{tabular}{ccc}
\hline \hline TO $\left(\mathrm{cm}^{-1}\right)$ & LO $\left(\mathrm{cm}^{-1}\right)$ & $\Gamma\left(\mathrm{cm}^{-1}\right)$ \\
\hline 69 & 70 & 21 \\
141 & 200 & 10 \\
249 & 275 & 7 \\
316 & 345 & 12 \\
345 & 379 & $?$ \\
382 & 459 & 20 \\
462 & 470 & 13 \\
591 & 659 & 18 \\
660 & 866 & 29 \\
\hline \hline
\end{tabular}

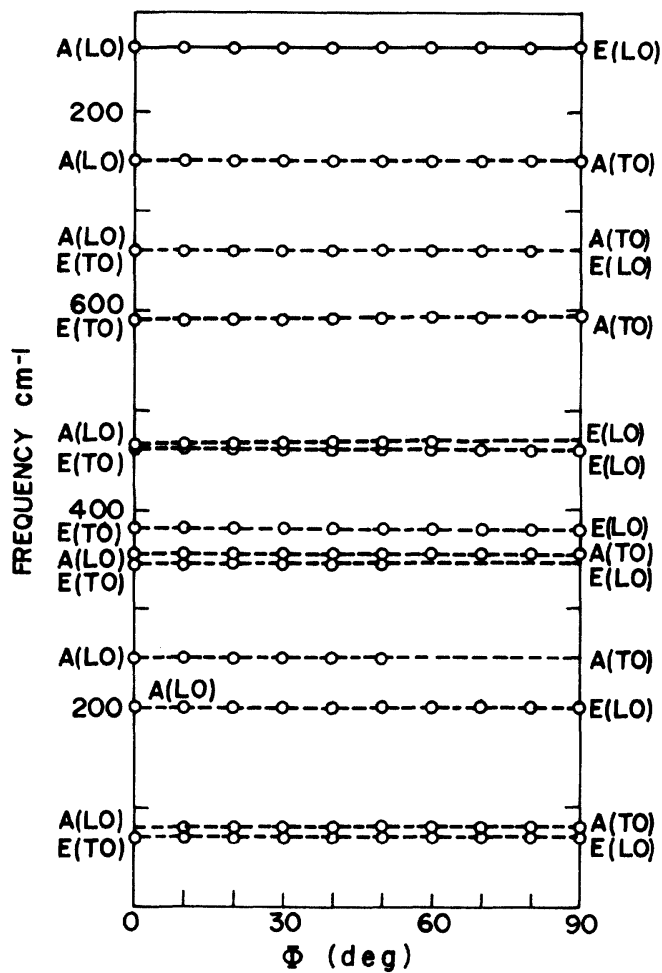

FIG. 13. Oblique phonon dispersion curves of the least dispersive modes in $\mathrm{LiTaO}_{3}$.

$$
\text { A-TO A, -LO E-TO E-LO }
$$
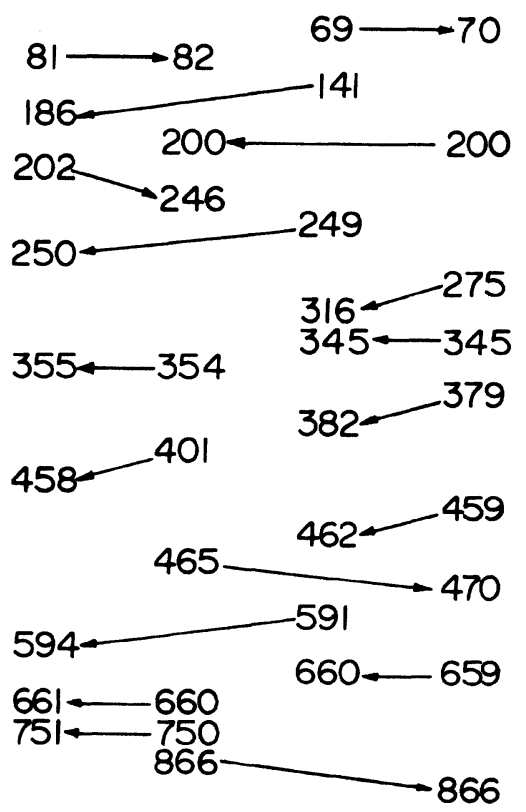

FIG. 14. Numerical map of the oblique phonon branches in $\mathrm{LiTaO}_{3}$. 
disperses with the $E(\mathrm{LO})$ at the same frequency.

The $E$ oblique phonons spectra are shown in Fig. 12 for five arbitrary angles taken at $X(Z Y) X+\Delta \Phi$ up to $500 \mathrm{~cm}^{-1}$. All the phonons that change character to $A_{1}$ in the oblique dispersion have been discussed above, while the ones having dispersion keeping the same character can easily be seen in the figure.

The experimental points of the oblique-phonon dispersion, for both $A_{1}$ and $E$, are shown in Figs. 6 and 13. Figure 14 provides also a numerical map showing the branches.

The oblique phonon dispersion measurements are very helpful to complete the phonon assignment for both TO and LO's and turns out to be a very accurate method to determine the exact phonon frequencies, especially for cases where the cross section is very small and therefore of difficult detection.

To conclude this paper, we want to comment on the previous assignments made by Kaminow and Johnston ${ }^{7.16}$ and Barker et al. ${ }^{8}$ In Refs. 7 and 16 four phonons were assigned as $A_{1}$ and they do coincide with four of ours (see Table II), but with respect to the $E$ phonons there are three of them (see Table I) which are in striking divergence with the oblique phonon dispersion; the $E(\mathrm{LO})$ at 163 $\mathrm{cm}^{-1}$ has never been observed by us and the $E$ (TO) at $206 \mathrm{~cm}^{-1}$ and $E(\mathrm{LO})$ at $248 \mathrm{~cm}^{-1}$ will correspond to ours if we exchange TO by $\mathrm{LO}$ and vice versa.

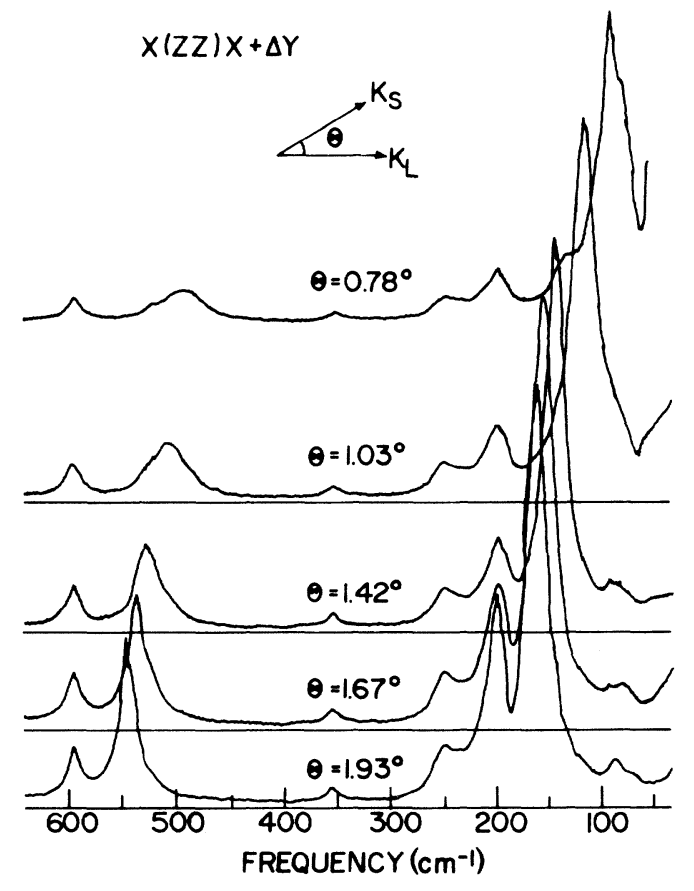

FIG. 15. $A_{1}$ polariton spectra in $\mathrm{LiTaO}_{3}$.

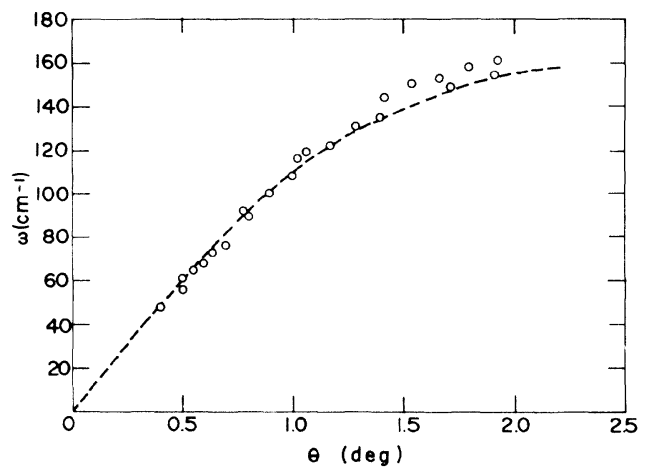

FIG. 16. Lowest $A_{1}$ polariton as a function of $\theta$. The circles are the experimental points and the dashed line is just a visual guide.

Barker et al..$^{8}$ used six oscillators (Table IV) to fit the infrared reflectivity parallel to $Z$ though only four were assigned as fundamental. They are more or less the same assigned by Kaminow and Johnston. The extra features along $Z$ are: TO's at 657 and $760 \mathrm{~cm}^{-1}$, and LO's at 625 and 760 $\mathrm{cm}^{-1}$. The modes at $760 \mathrm{~cm}^{-1}$ were assigned as $A_{1}$ type by us, while the mode at $657 \mathrm{~cm}^{-1}$ must correspond to our $A_{1}$ at $660 \mathrm{~cm}^{-1}$. The mode at 625 is just nonexistent in our data. Along the perpendicular direction, they used 13 oscillators to fit their spectrum of which nine are regarded as fundamental, namely, 142, 165, 215, 238, 253, 316, 375, 462 , and $594 \mathrm{~cm}^{-1}$. The main divergences with our assignment lies on the ones at 165, 215, and $238 \mathrm{~cm}^{-1}$. We cannot support those phonons since the first one is in contradiction with our obliquephonon data and of the other two we have no hints in the spectra. For the extra features in the perpendicular direction, we have no comments: it is just too much.

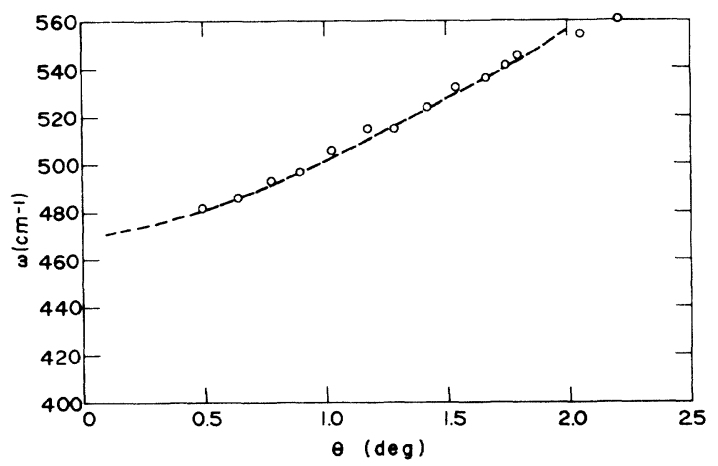

FIG. 17. Highest $A_{1}$ polariton. The circles are the experimental points and the dashed line is just a visual guide. 


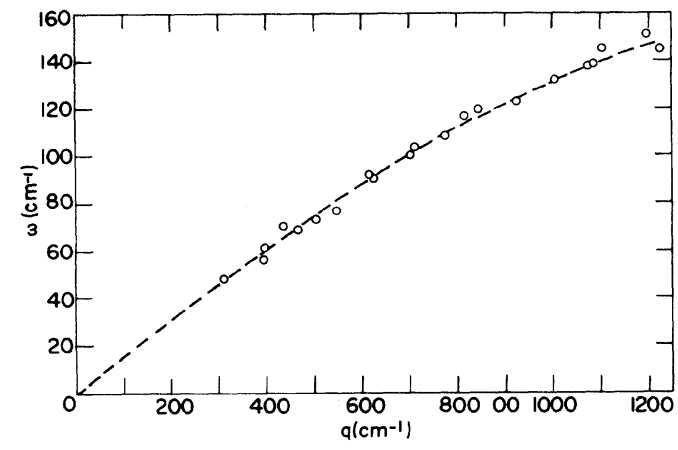

FIG. 18. Dispersion curve of the lowest $A_{1}$ polariton.

\section{POLARITONS}

As was discussed in Sec. II, only four $A_{1}$ phonons are allowed by group theory, if we consider the crystal as having a $C_{3 v}$ symmetry. Instead, we have found at least nine phonons belonging to this $A_{1}$ symmetry and attributed this to some lattice disorder, lowering the real symmetry to $C_{3}$, where nine $A_{1}$ modes are expected. Only two out of nine showed an appreciable change in frequency as a function of $q$. Several spectra are shown in Fig. 15, corresponding to angles between $2^{\circ}$ and

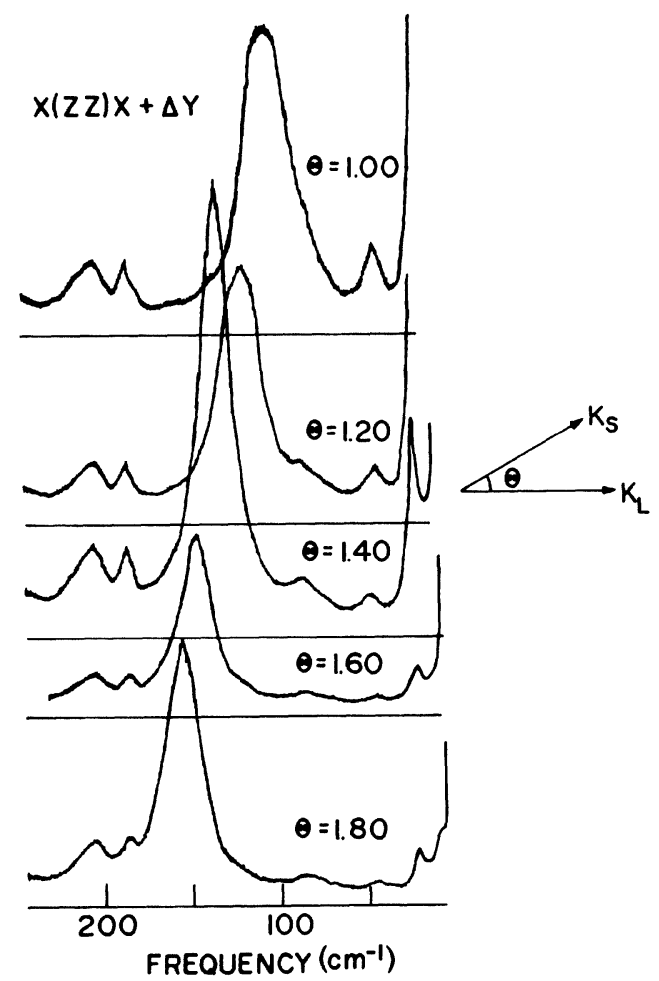

FIG. 19. Lowest $A_{1}$ polariton spectra in $\mathrm{LiTaO}_{3}$. Medium angles.

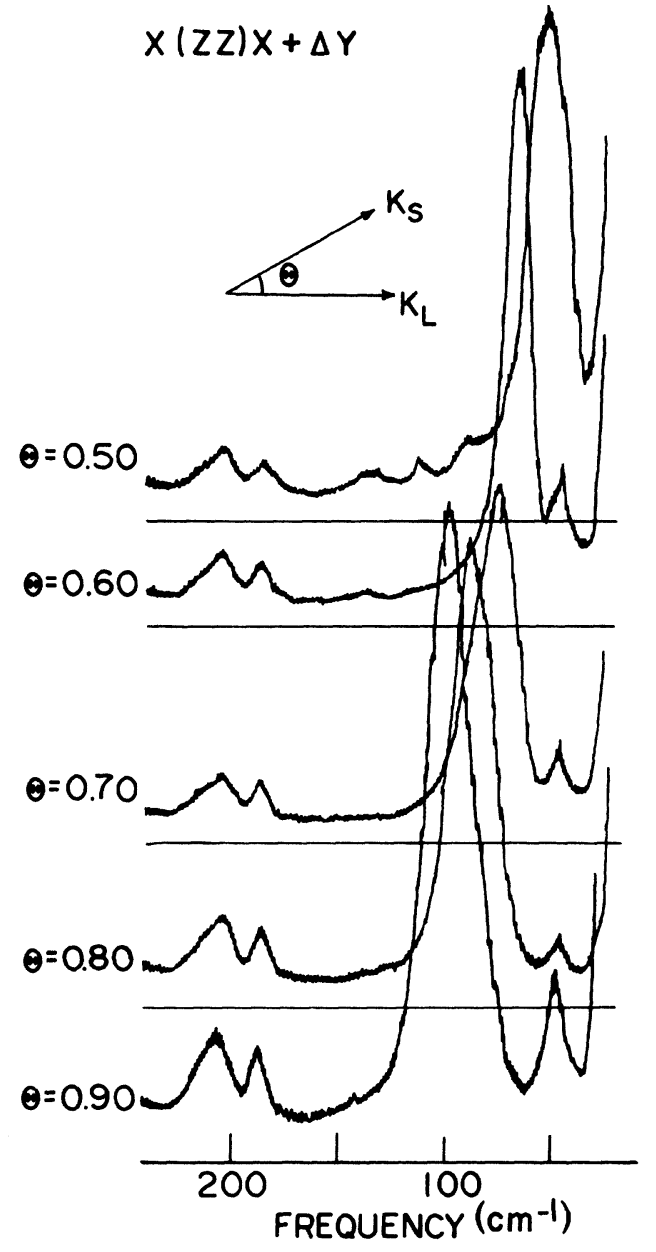

FIG. 20. Lowest $A_{1}$ polariton spectra in $\mathrm{LiTaO}_{3}$. Small angles.

$0.7^{\circ}$, taken at room temperature. Figures 16 and 17 show the experimental curves for both polaritons as a function of $\theta$, the angle between incident and scattered lights. From the momentum conservation law we can evaluate the magnitude of the wave vector $q$ [Fig. 2(b)] for any given $\theta$, and obtain the polariton dispersion curve. Since the higher dispersion curve gives no information about the static dielectric constant, we considered only the lower dispersion curve shown in Fig. 18. From its slope at zero frequence, we obtain the phonon contribution to the low-frequency dielectric constant, using expression (4). Doing that we obtain

$$
\epsilon_{0}=\left(\frac{\partial q}{\partial \omega}\right)_{q=0}^{2}=44
$$

which is in very good agreement with the result obtained by applying the Lyddane-Sachs-Teller relation in Sec. IV (48) and with the measurement 
done by Yamada et $a l .{ }^{18}$ using an admittance bridge (46). We want to emphasize that the phonon dispersing to zero in the polariton is the second one $\left(186 \mathrm{~cm}^{-1}\right)$ instead of the lower-frequency phonon $\left(81 \mathrm{~cm}^{-1}\right)$. There is a tendency to believe that the polariton going to zero is the lowest one, with no crossing feature. On the other hand, the almost vanishing split LO-TO in the lowest phonon (81 and $82 \mathrm{~cm}^{-1}$, respectively) must create a very weak electric field and as a consequence, a very weak phonon-lattice coupling, which favors the crossing to occur. If we take a look at Figs. 19 and 20, where we show in detail the lower polariton spectra down to $0.5^{\circ}$, it seems clear that the energy associated with the polariton is kept more or less constant while it disperses through the phonon at $82 \mathrm{~cm}^{-1}$. There is no sharp change in its intensity, which would be the case if it were to stay there and the dispersion to be continued by the lowest phonon, since its cross section is much smaller.
* Permanent address: Universidade Federal de Minas Gerais, Departamento de Física, Belo Horizonte, MG, Brasil.

${ }^{1}$ B. T. Matthias and J. P. Remeika, Phys. Rev. $\underline{76}, 1886$ (1949).

${ }^{2}$ P. V. Lenzo, E. H. Turner, E. G. Spencer, and A. A. Ballman, Phys. Lett. 8,81 (1966).

${ }^{3}$ S. C. Abrahams and J. L. Bernstein, J. Phys. Chem. Solids 28, 1685 (1967); A. S. Barker, Jr. and R. Loudon, Phys. Rev. 158, 433 (1967); H. D. Megaw, Acta Crystallogr. A 24, 583 (1968).

${ }^{4}$ S. C. Abrahams, W. C. Hamilton, and A. Siqueira, J. Phys. Chem. Solids 28, 1693 (1967).

${ }^{5}$ A. A. Ballman, H. J. Levinstein, C. D. Capio, and H. Brown, J. Am. Ceram. Soc. 50, 657 (1967); A. A. Ballman and H. Brown, J. Cryst. Growth 1 , 311 (1967); J. C. Bergman, A. Ashkin, A. A. Ballman, J. M. Dziedzic, H. J. Levinstein, and R. G. Smith, Appl. Phys. Lett. 12, 92 (1968).

${ }^{6}$ The results of the high-temperature measurements will be published elsewhere.

${ }^{7}$ I. P. Kaminow and W. D. Johnston, Jr., Phys. Rev. 160 , 519 (1967).
${ }^{8}$ A. S. Barker, Jr., A. A. Ballman, and J. A. Ditzenberger, Phys. Rev. B 2, 4233 (1970).

${ }^{9}$ R. Loudon, Adv. Phys. 13,423 (1964).

${ }^{10}$ E. B. Wilson, Jr., J. C. Decius, and Paul C. Cross, Molecular Vibrations (McGraw-Hill, New York, 1955), p. 334.

${ }^{11}$ W. Cochran and R. A. Cowley, J. Phys. Chem. Solids 23, 447 (1962).

${ }^{12} \mathrm{~K}$. Huang, Proc. R. Soc. Lond. 208, 352 (1951).

${ }^{13}$ C. M. Hartwig, D. L. Rousseau, and S. P. S. Porto, Phys. Rev. 188, 1328 (1969).

${ }^{14}$ W. S. Otaguro, E. Wiener-Avnear, C. A. Argüello, and S. P. S. Porto, Phys. Rev. B 4, 4542 (1971).

${ }^{15}$ E. Burstein, S. Ushioda, A. Pinczuk, and J. Scott, in Proceedings of the International Conference on Light Scattering Spectra in Solids, New York University, 1968, edited by G. B. Wright (Springer-Verlag, New York, 1969).

${ }^{16}$ W. D. Johnston, Jr. and I. P. Kaminow, Phys. Rev. 168, 1045 (1968).

${ }^{17}$ W. L. Bond, J. Appl. Phys. 36, 1674 (1965).

${ }^{18} \mathrm{~T}$. Yamada, N. Niizeki, and $\mathrm{H}$. Toyoda, Jpn. J. Appl. Phys. $\underline{7}, 292$ (1968). 